\title{
Interactive Effects of Dementia Severity and Comorbidities on Medicare Expenditures
}

\author{
Carolyn W. Zhu ${ }^{\mathrm{a}, \mathrm{b}, *}$, Stephanie Cosentino ${ }^{\mathrm{c}}$, Katherine A. Ornstein ${ }^{\mathrm{a}}$, Yian $\mathrm{Gu}^{\mathrm{c}}$, \\ Howard Andrews ${ }^{\mathrm{d}}$ and Yaakov Stern ${ }^{\mathrm{c}}$ \\ ${ }^{a}$ Department of Geriatrics and Palliative Medicine, Icahn School of Medicine at Mount Sinai, New York, \\ $N Y, U S A$ \\ ${ }^{\mathrm{b}}$ James J Peters VA Medical Center, Bronx, NY, USA \\ ${ }^{\mathrm{c} C o g n i t i v e ~ N e u r o s c i e n c e ~ D i v i s i o n ~ o f ~ t h e ~ D e p a r t m e n t ~ o f ~ N e u r o l o g y, ~ T a u b ~ I n s t i t u t e ~ f o r ~ R e s e a r c h ~ o n ~ A l z h e i m e r ' s ~}$ \\ Disease and the Aging Brain, Columbia University Medical Center, New York, NY, USA \\ ${ }^{\mathrm{d}}$ Department of Biostatistics, Mailman School of Public Health, Columbia University, New York, NY, USA
}

Accepted 13 January 2017

\begin{abstract}
.
Background: Few studies have examined how dementia and comorbidities may interact to affect healthcare expenditures. Objective: To examine whether effects of dementia severity on Medicare expenditures differed for individuals with different levels of comorbidities.

Methods: Data are drawn from the Washington Heights-Inwood Columbia Aging Project (WHICAP). Comprehensive clinical assessments of dementia severity were systematically carried out at $\sim 18$ month intervals. Dementia severity was measured by Clinical Dementia Rating (CDR). Comorbidities were measured by a modified Elixhauser comorbidities index. Generalized linear models examined effects of dementia severity, comorbidities, and their interactions on Medicare expenditures (1999-2010).

Results: At baseline, 1,280 subjects were dementia free $(\mathrm{CDR}=0,66.4 \%), 490$ had very mild dementia $(\mathrm{CDR}=0.5,25.4 \%)$, 108 had mild dementia $(C D R=1,5.6 \%)$, and 49 had moderate/severe dementia $(C D R=2 / 3,2.5 \%)$. Average annual Medicare expenditures for individuals with moderate/severe dementia were more than twice as high as those who were dementia free $(\mathrm{CDR}=0: \$ 9,108, \mathrm{CDR}=0.5 / 1: \$ 11,664, \mathrm{CDR} \geq 2: \$ 19,604, p<0.01)$. Expenditures were approximately 10 times higher among those with $\geq 3$ comorbidities than among those with no comorbidities $(\$ 2,612$ for those with no comorbidities, to $\$ 6,109$ for those with $1, \$ 10,656$ for those with 2 , and $\$ 30,244$ for those with $\geq 3$ comorbidities, $p<0.001$ ). Dementia severity was associated with higher expenditures, but comorbidities were the most important predictor of expenditures. We did not find strong interaction effects between number of comorbidities and dementia severity.

Conclusions: Increasing dementia severity and higher comorbidities are associated with higher Medicare expenditures. Care of individuals with dementia should focus on management of comorbidities.
\end{abstract}

Keywords: Health expenditure, Medicare, economics, comorbidities, longitudinal analysis

\section{INTRODUCTION}

\footnotetext{
${ }^{*}$ Correspondence to: Carolyn W. Zhu, PhD, Department of Geriatrics \& Palliative Medicine, Icahn School of Medicine at Mount Sinai and JJP VA Medical Center, 130 West Kingsbridge Road, Bronx NY 10468, USA. Tel.: +1 718584 9000/Ext. 6146; Fax: +1 718741 4211; E-mail: carolyn.zhu@mssm.edu.
}

Alzheimer's disease (AD) and related dementias are a major public health challenge in the US. In 2015, direct costs of caring for those with Alzheimer's disease (AD), the most prevalent form of dementia, are estimated to be $\$ 226$ billion, with half of the 
costs borne by Medicare [1]. Average annual cost of care for each AD patient is estimated to be between $\$ 41,689$ and $\$ 56,290$, more than twice the cost of care for individuals without dementia [1,2]. Among individuals with dementia, prevalence of coexisting conditions is high. Some of the most common conditions, including hypertension, diabetes, congestive heart failure, coronary heart diseases, have been reported to be higher in dementia patients compared to others without dementia, although rates of other conditions such as chronic kidney disease and cancer have been reported to be lower [1-19].

Comorbidities in patients with dementia present particular challenges for their care. Dementia may complicate a patient's clinical management, and some conditions may exacerbate the progression of dementia, increasing cost of care. On the other hand, clinicians may be more reluctant to treat patients with dementia because of difficulties in communicating with patient or obtaining patient cooperation, or a patient with dementia may not be considered a good candidate for aggressive interventions and cost of care may actually be lower than that of another patient with otherwise similar conditions. The severity of dementia and number of comorbidities also may affect cost of care differently. While dementia and comorbidities have been shown to be independently associated with higher healthcare use and costs $[2,11$, 20-24], fewer studies have examined how dementia and comorbidities may interact to affect healthcare use and expenditures. In a study that reported Medicare claims based dementia diagnosis were associated with higher rates of all-cause and preventable hospitalizations, the magnitude of the effect of dementia was lower in patients with higher comorbidities [21], suggesting that the effects of dementia on hospitalization are modified by comorbidity burden. But the study did not examine how this interaction ultimately impact cost. Furthermore, dementia severity also was not considered in the study. Only one early study reported that while dementia severity and comorbidities were independently associated with higher cost of care, dementia severity did not have an interactive effect with comorbidities on cost [25].

In this study, we take advantage of a unique longitudinal study that prospectively followed a cohort of Medicare beneficiaries for whom comprehensive assessments were systematically and frequently carried out, and for whom expenditures data are available from Medicare claims. The main goal of this study was to explore the relationship between dementia severity and comorbidities and examine whether cost effects of dementia severity differed for individuals with different levels of comorbidities. We extend the literature by using dementia diagnoses based on clinical consensus instead of claims-based diagnoses. The latter have been shown to underestimate or misidentify dementia and provide little information on disease severity [26-28].

\section{METHODS}

\section{Participants}

Participants were drawn from the Washington Heights-Inwood Columbia Aging Project (WHI CAP), a multiethnic, population-based, prospective study of cognitive aging of Medicare beneficiaries age 65 and older residing in northern Manhattan. Lists of all Medicare or Medicaid recipients in the study area were provided by the Centers for Medicare and Medicaid Services (CMS) at the beginning of study enrollment in 1992. A total of 2,125 subjects were recruited. A "refreshment" cohort of 2,183 additional participants was formed in 1999 using generally similar methods, with several exceptions: new lists of beneficiaries were obtained but those drawn into the 1992 cohort were excluded. The original list of names was divided into six strata based on age (65-74 years and 75 years and older) and ethnic group. Ethnic groups (Hispanics, non-Hispanic blacks, non-Hispanic whites) were categorized using CMS data supplemented by 1990 US Census files that included a Hispanic surname. These strata were further subdivided into subsamples so that the distributions by age and ethnic group within each subsample were similar. This provided a means to ensure equal representation of the community during the initial assessment of participants. Based on the distributions within the subsamples, the proportion of individuals within age and ethnic group who participated in the study did not differ significantly from the source population. Detailed descriptions of study methodology have been reported previously $[29,30]$. At the time of study entry, each subject underwent an in-person interview of general health and functional ability, followed by a standardized assessment including medical history, physical and neurological examination, and a neuropsychological battery. After the baseline assessment, subjects were followed at approximately 18-month intervals with similar assessments till death, or lost to follow up, whichever is earlier. Evaluations were conducted in either 


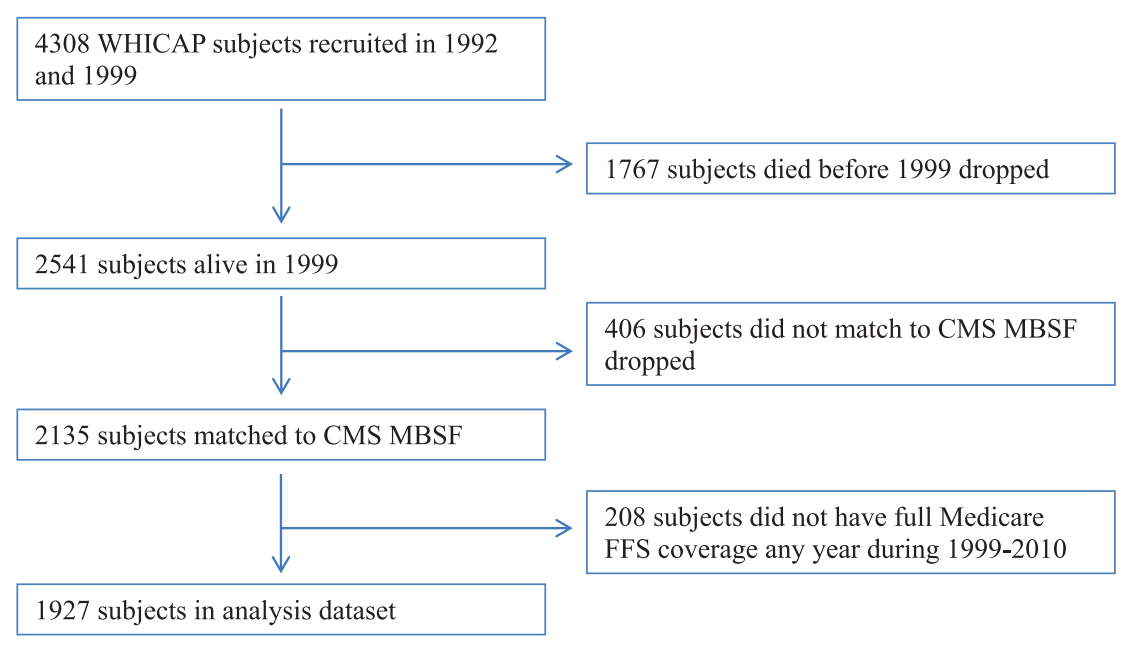

Fig. 1. Sample selection. CMS, Centers for Medicare and Medicaid Services; MBSF, Medicare Beneficiary Summary file; FFS, Fee for Service.

English or Spanish, based on the participant's primary language or preference. Recruitment, informed consent and study procedures were approved by the Institutional Review Boards of Columbia Presbyterian Medical Center and Columbia University Health Sciences, the New York State Psychiatric Institute, and the CMS Privacy Board. Written informed consent was obtained from all subjects.

To obtain data on Medicare utilization and expenditures, individuals were matched to the Medicare Beneficiary Summary file (MBSF) using social security number and Medicare beneficiary ID. The study sample included individuals followed from their first WHICAP visit or the beginning of Medicare data availability (January 1, 1999), and ended at death, end of study follow-up, or end of Medicare data availability (December 31, 2010). Specifically, 2,541 subjects who were alive in 1999 were considered for the current analysis. 406 subjects who did not match the CMS crosswalk file or who did not match the CMS MBSF were then dropped. Because data from individuals who were covered under Medicare managed care are incomplete in the Medicare claims, we followed CMS Chronic Condition Warehouse (CCW) guideline for full coverage definition and excluded observations from subjects who were not covered by Medicare fee-for-service (FFS) providers for 10 or more months during a calendar year (or had no more than 1 month which is not covered during the year of death if the subject died) [31]. The current analysis included 1,927 remaining subjects. Figure 1 summarizes our sample selection process. Baseline in the current analysis is defined to be the first calendar year in which a subject who had a WHICAP evaluation who also had full Medicare coverage.

\section{Dementia severity}

At each WHICAP assessment, diagnostic conferences were held by a group of neurologists, psychiatrists, and neuropsychologists using results from the neuropsychological battery as well as evidence of impairment in social or occupational function [29, 32]. A diagnosis of dementia was determined based on the Diagnostic and Statistical Manual of Mental disorders, revised Fourth Edition criteria. The type of dementia was subsequently determined. Diagnosis of probable or possible AD was made based on criteria outlined by the National Institute of Neurological and Communicative Disorders and Stroke-Alzheimer's Disease and Related Disorders Association. Dementia severity was measured by the Clinical Dementia Rating (CDR) [33]. The CDR has advantage of combining both function and cognitive manifestations of the disease, and is well standardized and segments in the disease into well-understood levels of severity.

\section{Medicare expenditures}

Medicare expenditures data were obtained from Medicare Standard Analytic Files (SAFs) and included all covered services (inpatient, outpatient, physician, durable medical equipment, skilled 
nursing, home health, and hospice care). Expenditures reflect actual payments to each beneficiary and were summarized annually by calendar year. Because WHICAP subjects were followed at 18 month intervals, we used WHICAP assessment dates as anchors and aligned Medicare expenditures to the calendar year. Medicare data in years in which there were no clinical assessment were excluded. Because all Medicare data during the year of death for those who died were observed, we did not annualize cost during the last year of life [34]. All expenditures were adjusted to $2012 \$$ using the medical care component of the Consumer Price Index [35].

\section{Comorbidities}

All ICD-9-CM diagnosis codes in all Medicare SAFs were used to identify comorbid conditions in the Elixhauser comorbdity index (range $=0-30$ ). To prevent over-estimation of the comorbidity when using physician or outpatient claims, we followed the literature and applied a rule-out algorithm to physician/outpatient claims, requiring occurrences of the diagnostic code on at least two different claims that are more than 30 days apart when identifying conditions in these claims [36, 37]. A modified Elixhauser comorbidities index during a year was constructed by summing all individual indicators (empirical range $=0-11$ ), excluding dementia. (Detailed codes for identifying each condition can be found in Quan et al. [37].) Because $90 \%$ of all subjects had 3 or fewer comorbidities at baseline, we grouped comorbidities into four categories: $0,1,2$, and $3+$ comorbidities $[37,38]$.

\section{Other clinical and socio-demographic characteristics}

Individuals' disability, which may or may not be related to dementia, was measured by difficulties performing various activities of daily living (ADL) using the Blessed Functional Activity Scale [39]. Individual items were summed (range $=0-9$ ) and grouped into the following categories: $\mathrm{ADL}=0,1$, and $\geq 2$ based on empirical distributions. Extrapyramidal signs (EPS) were assessed using a modified Unified Parkinson's Disease Rating Scale, whose inter-rater reliability has already been established [40]. A total EPS score was computed and dichotomized into $<2$ (slight or none) versus $\geq 2$ (moderate or severe). Drug-induced EPS were excluded from the analyses.
The socio-demographic characteristics were included a priori in multivariate analyses based on known associations between these predictors and healthcare spending: age, sex, ethnicity, years of education, marital status, and Medicaid eligibility as a proxy for socioeconomic status. Because death is strongly associated with healthcare expenditures, we included an indicator for death during the year.

\section{Analysis}

Baseline socio-demographic and clinical characteristics by dementia severity were compared using chi-square test for categorical variables and Kruskal-Wallis test for continuous variables. We also compared Medicare expenditures by dementia severity and comorbidities.

There are a number of well-known methodological challenges in analyzing cost data, including (1) non-negative costs, (2) excess zeroes from individuals not using healthcare services during a particular period, (3) heavy right tails, and (4) substantial right-skewness [41]. Several categories of analytical approaches have been developed to tackle these issues [42]. In the current sample, the vast majority of individuals used Medicare services at some time during the study period, so excess zeroes are less of a concern. We considered several methodologies that are more suitable for our sample, including ordinary least squares regression (OLS), methods following transformation of data, generalized linear models (GLMs), and parametric models based on skewed distributions outside the GLM family. Unless the dataset is sufficiently large, OLS typically results in inconsistent or inefficient estimates [43]. In order to account for the problem of skewness, methods following transformation of data (most often log transformation) have been commonly used. However, log costs estimates are not of interest for decision makers, and retransformation is needed for estimates to be re-interpreted to the original cost scale. More recently, generalized linear models (GLM) have been increasingly used as an improved method of analyzing cost. In the current analysis, our analytic problem is further complicated by the longitudinal nature of the data. In theory, generalized linear mixed models (or GLMMs) are an extension of GLMs that can be used in this setting. However, it is not yet clear how the characteristics of the cost data are dealt with in this model. Because multiple observations may be included as subjects were followed over time, cluster robust standard errors were estimated by integrating subject 
ID as a cluster term. We examined the appropriateness of distributional family and link functions using modified Park test and chose GLM with gamma family and log link as our estimation method. Our main independent variables are comorbidities, dementia severity, and their interaction terms. We hypothesize that the main effects of comorbidities and dementia severity are both positive. Our main interest is the coefficient on their interaction terms. If the interaction term is statistically non-significant, this indicates no modifying effects of dementia severity and comorbidities. If it is positive, this indicates that increasing dementia severity and higher comorbidities are associated with increasingly higher cost of care. If it is negative, this indicates that cost of care in individuals with more comorbidities is lower in the presence of dementia. Because of the small proportions of the sample with $\mathrm{CDR}=3$ at any assessment interval, we combined it with the $\mathrm{CDR}=2$ group. We also combined $\mathrm{CDR}=0.5$ and $\mathrm{CDR}=1$ groups as exploratory analysis showed little difference in costs between these two groups. An individual's CDR status could change over time. Control variables included disability, EPS, age, gender, race/ethnicity, education, marital status, Medicaid eligibility, died during the year, and indicators for year. Except for gender, race/ethnicity, and education, all other variables are included as time-varying covariates.

All analyses were performed using Stata 13.0. Statistical significance was set a priori at $p<0.05$.

\section{Sensitivity analyses and other considerations}

\section{Alternative measures of comorbidity}

We constructed an unweighted modified Charlson comorbidity scores based on Medicare claims [37], and also a modified weighted Charlson index based on WHICAP survey questions as alternative measures of comorbidity [44, 45]. Estimated results using these comorbidity measures were substantively similar to those derived from using the Elixhauser index. Complete estimation results using all three comorbidity indices can be obtained from the authors.

\section{Multiple interactions}

The interactions between dementia severity, comorbidities, and functional limitations are likely to be complex. Dementia severity and functional limitation, as well as functional limitations and comorbidity are related and are important determinants of cost of care [20]. To examine this issue, we first tested correlations between these variables. Although highly signification, the magnitude of the correlation across all assessments between functional limitations and comorbidities (rho $=0.119)$ and between comorbidities and CDR (rho $=0.084)$ in this sample were fairly low (both $p<0.001$ ). The correlation between functional limitations and CDR was higher ( rho $=0.438$, $p<0.001$ ). We then explored estimation models that included these three-way interactions. Results did not show significant interactions between functional limitations and dementia severity. All of the threeway interactions were statistically non-significant. Because our focus was to examine the relationship between dementia severity and comorbidities, we included functional disability measure as a control variable in our analyses.

\section{RESULTS}

\section{Sample characteristics at baseline}

The mean age of the sample at baseline was $78.0 \pm 7.0$ years. The sample was $67.5 \%$ women, 43.5\% Hispanic (mainly of Caribbean ancestry) and 24.9\% African American. At baseline, 1,280 subjects were dementia free $(C D R=0,66.4 \%), 490$ had very mild dementia $(\mathrm{CDR}=0.5,25.4 \%), 108$ had mild dementia ( $\mathrm{CDR}=1,5.6 \%)$, 33 had moderate dementia $(C D R=2,1.7 \%)$, and the rest 16 had severe dementia $(C D R=3,0.8 \%)$ (Table 1$)$. At the last assessment, $57.4 \%$ of the subjects had $\mathrm{CDR}=0$, $25.2 \%$ had $\mathrm{CDR}=0.5,9.4 \%$ had $\mathrm{CDR}=1,8.0 \%$ had $\mathrm{CDR}=2$ or worse. Individuals with more severe dementia were older, more likely to be female, and had more functional limitations and extrapyramidal signs than those with no or mild dementia. On average, individuals who were initially dementia free were followed in the WHICAP study for $3.4 \pm 3.2$ years $(2.2 \pm 2.7$ for those who were initially mildly demented, and $1.6 \pm 0.9$ for those who were initially with moderate/severe dementia). Average years of Medicare data contribution were similar across dementia severity groups $(2.2 \pm 1.2,2.2 \pm 1.1$, and $2.2 \pm 1.1$ years for those who were initially dementia free, with mild dementia, and with moderate/severe dementia, respectively). By the end of the study, $34.7 \%$ of those who were initially dementia free had died, as did $42.4 \%$ of those who were initially mildly demented, and $73.4 \%$ of those who were initially with moderate/severe dementia. 
Table 1

Sample characteristics at baseline by dementia severity

\begin{tabular}{|c|c|c|c|c|}
\hline & $\begin{array}{l}\text { No dementia } \\
\mathrm{CDR}=0\end{array}$ & $\begin{array}{c}\text { Mild dementia } \\
\mathrm{CDR}=0.5 / 1\end{array}$ & $\begin{array}{c}\text { Moderate/Severe dementia } \\
\mathrm{CDR} \geq 2\end{array}$ & $p$-value \\
\hline$\overline{\mathrm{N}}$ & 1770 & 108 & 49 & \\
\hline Age, mean (sd) & $77.4(6.7)$ & $83.8(7.0)$ & $85.3(7.1)$ & $* * *$ \\
\hline Female $(\%)$ & 66.4 & 77.8 & 85.7 & $* * *$ \\
\hline Ethnicity (\%) & & & & $* * *$ \\
\hline White non-Hispanic & 32.0 & 11.1 & 14.3 & \\
\hline Black non-Hispanic & 24.9 & 25.9 & 24.5 & \\
\hline Hispanic & 41.9 & 63.0 & 59.2 & \\
\hline Years of education, mean (sd) & $10.4(4.8)$ & $6.6(4.6)$ & $7.1(4.8)$ & $* * *$ \\
\hline Marital status $(\%)$ & & & & $* * *$ \\
\hline Married & 31.6 & 20.0 & 25.5 & \\
\hline Widowed & 36.1 & 49.5 & 61.7 & \\
\hline Never married & 13.0 & 10.5 & 4.3 & \\
\hline Divorced/separated & 19.2 & 20.0 & 8.5 & \\
\hline Medicaid eligible (\%) & 34.5 & 61.1 & 49.0 & $* * *$ \\
\hline Number of ADL limitations, mean (sd) & $0.2(0.7)$ & $1.1(1.7)$ & $4.6(3.2)$ & $* * *$ \\
\hline $0(\%)$ & 90.4 & 52.8 & 18.8 & \\
\hline $1-2(\%)$ & 7.6 & 30.2 & 10.4 & \\
\hline$\geq 3(\%)$ & 2.0 & 17.0 & 70.8 & \\
\hline Total EPS score, mean (sd) & $0.9(1.9)$ & $2.6(3.0)$ & $6.7(6.2)$ & $* * *$ \\
\hline $\mathrm{EPS}=0(\%)$ & 66.4 & 34.6 & 21.7 & \\
\hline $\mathrm{EPS}=1(\%)$ & 32.0 & 55.1 & 58.7 & \\
\hline $\mathrm{EPS} \geq 2(\%)$ & 1.6 & 10.3 & 19.6 & \\
\hline Modified Elixhauser score, mean (sd) & $1.1(1.7)$ & $1.7(1.9)$ & $2.2(2.1)$ & $* * *$ \\
\hline $0(\%)$ & 52.9 & 36.1 & 32.7 & \\
\hline $1(\%)$ & 16.9 & 19.4 & 10.2 & \\
\hline $2(\%)$ & 13.4 & 18.5 & 14.3 & \\
\hline$\geq 3(\%)$ & 16.8 & 25.9 & 42.9 & \\
\hline Years of follow up, mean (sd) & $3.4(3.2)$ & $2.2(2.7)$ & $1.6(0.9)$ & $* * *$ \\
\hline Died at end of study (\%) & 34.7 & 42.4 & 73.4 & $* * *$ \\
\hline
\end{tabular}

The modified Elixhauser index was $1.2 \pm 1.7$ for the entirely sample, $1.1 \pm 1.7$ in those without dementia, $1.7 \pm 1.9$ in those with mild dementia, and $2.2 \pm 2.1$ in those with moderate/severe dementia. The most common conditions included hypertension $(34.5 \%)$, diabetes (16.3\%), cardiac arrhythmia (7.3\%), chronic pulmonary disease $(7.3 \%)$, and congestive heart failure $(6.9 \%)$. Prevalence of these comorbidities was higher in those with more severe dementia.

\section{Dementia severity, comorbidities, and Medicare expenditures}

Unadjusted expenditures by dementia severity and comorbidities across all assessments are shown in Table 2. Average annual per capita Medicare expenditures for individuals with moderate/severe dementia were more than twice as high as those who did not have dementia $(\$ 9,108$ for those with $C D R=0$, $\$ 11,664$ for those with $\mathrm{CDR}=0.5 / 1$, and $\$ 19,604$ for those with $\mathrm{CDR} \geq 2, p<0.01)$. Within each comorbidities group, differences in mean costs across dementia severity groups remain statistically significant (all $p<0.001$ ), but median costs were similar across dementia severity groups.

Comorbidities were strongly associated with expenditures. For the entire sample, average per capita expenditures were approximately 10 times higher among those with $\geq 3$ comorbidities than among those with no comorbidities $(\$ 2,612$ for those with no comorbidities, to $\$ 6,109$ for those with 1 , $\$ 10,656$ for those with 2 , and $\$ 30,244$ for those with $\geq 3$ comorbidities, $p<0.001)$. Within each CDR level, differences in mean and median costs across comorbidity groups are statistically significant (all $p<0.001)$.

\section{Regression estimates on Medicare expenditures}

Estimated effects of dementia severity and comorbidities on Medicare expenditures are in Table 3. After controlling for other covariates, number of comorbidities continued to have the strongest effects on expenditures. Estimated coefficients were larger for higher comorbidities, suggesting larger increases 
Table 2

Unadjusted annual per capita expenditures by dementia severity and number of comorbidities

\begin{tabular}{|c|c|c|c|c|c|}
\hline & & \multirow[b]{2}{*}{ All Sample } & \multicolumn{3}{|c|}{ Dementia Severity } \\
\hline & & & $\begin{array}{l}\text { No Dementia } \\
\text { CDR }=0\end{array}$ & $\begin{array}{c}\text { Mild dementia } \\
\mathrm{CDR}=0.5 / 1\end{array}$ & $\begin{array}{c}\text { Moderate/Severe dementia } \\
\text { CDR } \geq 2\end{array}$ \\
\hline All Sample & $\begin{array}{l}\text { Mean } \\
\text { (sd) } \\
\text { Median } \\
\text { IQR }\end{array}$ & $\begin{array}{c}9,702 \\
(18,769) \\
2,889 \\
{[1131,8792]}\end{array}$ & $\begin{array}{c}9,108 \\
(18,029) \\
2,782 \\
{[1,104,8,076]}\end{array}$ & $\begin{array}{c}11,664 \\
(18,570) \\
4,441 \\
{[1,300,12,075]}\end{array}$ & $\begin{array}{c}19,604 \\
(29,377) \\
6,578 \\
{[1,545,24,036]}\end{array}$ \\
\hline $\begin{array}{l}\text { Modified El } \\
\quad 0\end{array}$ & $\begin{array}{l}\text { Mean } \\
\text { (sd) } \\
\text { Median } \\
\text { IQR }\end{array}$ & $\begin{array}{c}2,612 \\
(3,871) \\
1,623 \\
{[638,3,251]}\end{array}$ & $\begin{array}{c}2,589 \\
(3,729) \\
1,645 \\
{[642,3,251]}\end{array}$ & $\begin{array}{c}2,638 \\
(3,084) \\
1,579 \\
{[532,3,808]}\end{array}$ & $\begin{array}{c}3,310 \\
(7,813) \\
1,174 \\
{[638,2,472]}\end{array}$ \\
\hline 1 & $\begin{array}{l}\text { Mean } \\
\text { (sd) } \\
\text { Median } \\
\text { IQR }\end{array}$ & $\begin{array}{c}6,109 \\
(8,836) \\
2,996 \\
{[1,321,6,894]}\end{array}$ & $\begin{array}{c}6,090 \\
(8,648) \\
3,088 \\
{[1,351,7,072]}\end{array}$ & $\begin{array}{c}5,027 \\
(7,158) \\
2,632 \\
{[1,045,6,271]}\end{array}$ & $\begin{array}{c}10,016 \\
(17,258) \\
2,700 \\
{[1,310,8,776]}\end{array}$ \\
\hline 2 & $\begin{array}{l}\text { Mean } \\
\text { (sd) } \\
\text { Median } \\
\text { IQR }\end{array}$ & $\begin{array}{c}10,656 \\
(14,507) \\
5,173 \\
{[1,978,13,029]}\end{array}$ & $\begin{array}{c}10,599 \\
(14,651) \\
4,922 \\
{[1,913,13,091]}\end{array}$ & $\begin{array}{c}10,666 \\
(14,165) \\
6,484 \\
{[1,525,10,887]}\end{array}$ & $\begin{array}{c}11,814 \\
(12,497) \\
8,804 \\
{[2,645,18,153]}\end{array}$ \\
\hline$\geq 3$ & $\begin{array}{l}\text { Mean } \\
\text { (sd) } \\
\text { Median } \\
\text { IQR }\end{array}$ & $\begin{array}{c}30,244 \\
(31,490) \\
19,058 \\
{[9,165,42,970]}\end{array}$ & $\begin{array}{c}29,735 \\
(31,541) \\
18,729 \\
{[8,578,41,710]}\end{array}$ & $\begin{array}{c}27,858 \\
(25,488) \\
17,362 \\
{[10,777,42,846]}\end{array}$ & $\begin{array}{c}37,736 \\
(36,201) \\
23,915 \\
{[10,103,58,964]}\end{array}$ \\
\hline
\end{tabular}

IQR, interquartile range.

Table 3

Regression estimates on annual per capita Medicare expenditures: effects of dementia severity and number of comorbidities

\begin{tabular}{|c|c|c|c|c|c|c|}
\hline \multirow[b]{2}{*}{ Variables } & & \multicolumn{2}{|c|}{$\begin{array}{c}\text { Annual } \\
\text { Expenditures }\end{array}$} & \multirow[b]{2}{*}{$p$-values } & \multirow{2}{*}{\multicolumn{2}{|c|}{$\begin{array}{l}\text { 95\% Confidence } \\
\text { Intervals } \\
\end{array}$}} \\
\hline & & Coeff. & (se) & & & \\
\hline \multirow[t]{4}{*}{ Comorbidities } & $\begin{array}{c}\text { Elixhauser }=0 \\
\quad(\text { reference })\end{array}$ & & & & & \\
\hline & Elixhauser $=1$ & 0.840 & $(0.059)$ & $<0.001$ & 0.721 & 0.959 \\
\hline & Elixhauser $=2$ & 1.432 & $(0.066)$ & $<0.001$ & 1.300 & 1.564 \\
\hline & Elixhauser $\geq 3$ & 2.394 & $(0.052)$ & $<0.001$ & 2.291 & 2.497 \\
\hline \multirow[t]{3}{*}{ CDR } & $\begin{array}{c}\mathrm{CDR}=0 \\
\text { (reference) }\end{array}$ & & & & & \\
\hline & $\mathrm{CDR}=0.5 / 1$ & 0.214 & $(0.100)$ & 0.032 & 0.014 & 0.414 \\
\hline & $\mathrm{CDR} \geq 2$ & 0.372 & $(0.189)$ & 0.048 & 0.005 & 0.750 \\
\hline \multirow[t]{3}{*}{ Interaction with $\mathrm{CDR}=0.5 / 1$} & Elixhauser $=1$ & 0.216 & $(0.175)$ & 0.217 & -0.134 & 0.566 \\
\hline & Elixhauser $=2$ & 0.328 & $(0.167)$ & 0.043 & -0.005 & 0.662 \\
\hline & Elixhauser $\geq 3$ & -0.136 & $(0.138)$ & 0.322 & -0.411 & 0.139 \\
\hline \multirow[t]{3}{*}{ Interaction with $\mathrm{CDR} \geq 2$} & Elixhauser $=1$ & 0.071 & $(0.407)$ & 0.862 & -0.743 & 0.884 \\
\hline & Elixhauser $=2$ & -0.068 & $(0.276)$ & 0.805 & -0.620 & 0.484 \\
\hline & Elixhauser $\geq 3$ & -0.301 & $(0.232)$ & 0.195 & -0.756 & 0.154 \\
\hline
\end{tabular}

Other variables included in the models were disability, EPS, age, gender, race/ethnicity, education, marital status, Medicaid eligibility, died during the year, and indicators for year.

in costs from higher comorbidities. Dementia severity was independently associated with higher expenditures. The interaction terms between dementia severity and number of comorbidities were mostly statistically non-significant.

Predicted expenditures by dementia severity and comorbidities using these estimated regression coefficients are shown in Fig. 2. Within each dementia severity group, expenditures were significantly higher for those with higher comorbidities. Specifically, in individuals without dementia, expenditures were predicted to range from $\$ 2,531 \pm 377$ in those without any comorbidities to $\$ 27,732 \pm 1,172$ in those with $3+$ comorbidities. In individuals with 


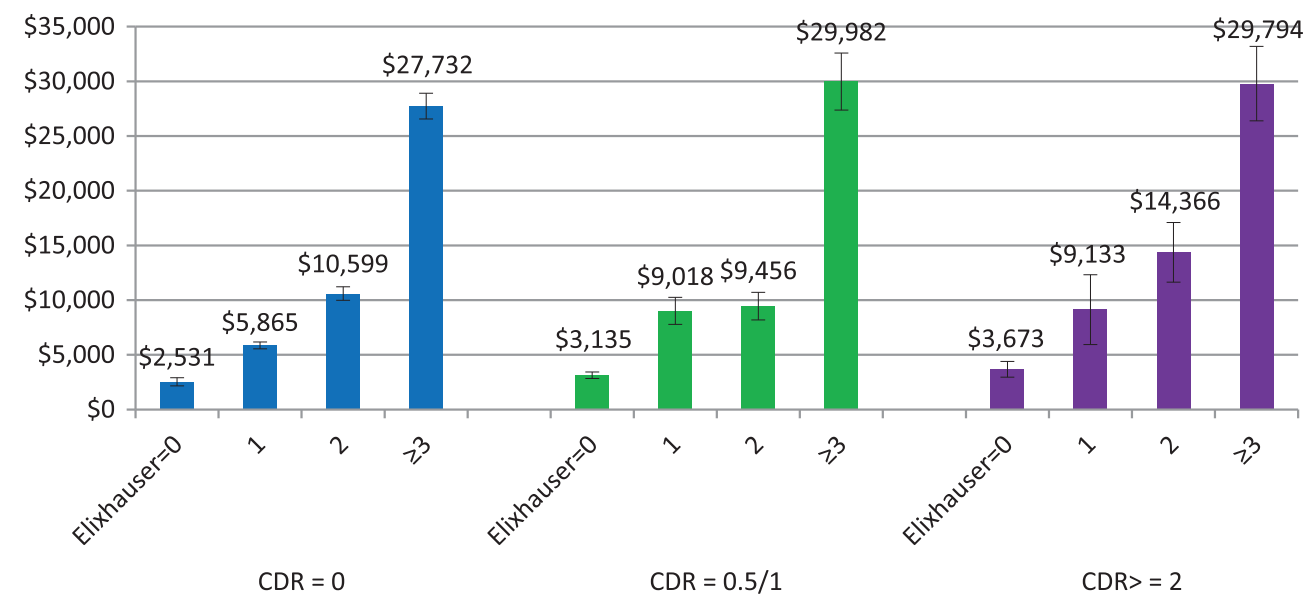

Fig. 2. Predicted mean expenditures by dementia severity and comorbidity groups. Vertical bars indicate estimated standard errors.

mild dementia, expenditures were predicted to range from $\$ 3,135 \pm 300$ for those without any comorbidities to $\$ 29,982 \pm 2,606$ for those with $3+$ comorbidities. In those with moderate/severe dementia, expenditures were predicted to range from $\$ 3,673 \pm 722$ for those without any comorbidities to $\$ 2,979 \pm 3,402$ for those with $3+$ comorbidities. Conversely, within each comorbidities group, predicted expenditures also were higher for higher dementia severity, but the magnitude of the effects was not as large.

Higher expenditures also were associated with higher functional limitations and extrapyramidal signs. Older age, being female, having higher education, and death during the year also were associated with higher expenditures (results are provided in Supplementary Table 1).

\section{DISCUSSION}

Existing studies on cost of dementia have often used claims-based dementia diagnosis, which have been shown to underestimate or misidentify dementia and provide little information on disease severity. In this study, we explored the relationship between dementia severity and comorbidities and examined their effects on Medicare spending in an ethnically diverse cohort of individuals for whom dementia was clinically diagnosed and dementia severity was systematically assessed over time. Epidemiologic studies that have both clinically assessed dementia and comprehensive cost data such as those from Medicare claims are rare. In combining the two sources of data, we aimed to avoid biases that may have been introduced in using claims based data only. To put our study in context, it is important to note several important differences in our study aside from how dementia was assessed. Most notably, our sample is almost two thirds minority, predominantly Hispanic, and was recruited from a defined geographic area. Caution should be exercised in generalizing our results to other populations. That said, our results showed that after controlling for other determinants of cost, dementia severity and comorbidities were both independently associated with higher costs. Consistent with existing studies that showed that comorbidities accounted for substantial proportion of healthcare use and costs [19], we found that comorbidities were the strongest drivers of higher costs of care. Similar to an early study which examined interactive effect of dementia severity and comorbidities on cost [25], we also did not find a substantial modifying effect of dementia severity and level of comorbidities on costs of care.

Multimorbidity has been shown to have substantial negative impact on a person's health and on the continuity of primary care [46, 47]. Our results show that in the context of dementia, this relationship remains. Several very large studies using administrative datasets reported that for some of the most prevalent comorbid conditions, costs for dementia patients were substantially higher compared to demographically matched control subjects $[5,9]$. In a set of secondary analyses, we estimated the effects of several common comorbid conditions on Medicare expenditures, e.g., hypertension, diabetes, and cardiac arrhythmia. Results showed these comorbidities were associated with higher expenditures, but interaction effect between individual comorbidities and 
dementia severity were not statistically significant. Aside from differences in sample characteristics, the statistical non-significance in our results possibly related to large differences in sample sizes between these studies. Healthcare delivery, research, and education often focus on individual diseases, but people with multimorbidity may have needs that are different from those with only one condition, and that need a broader approach in their care. Many models of care are focused on individual diseases and do not take into account the needs of those with multiple conditions $[48,49]$. With the growing number of older adults with dementia and multiple chronic conditions, understanding whether and how comorbidities and dementia severity may interact to affect healthcare costs are highly relevant for healthcare planning, allocation of resources, and development of strategies to improve care and manage costs. Our results also point to the importance of examining dementia severity instead of only presence of dementia. Many studies, especially those based on claims data, often only use a binary indicator of dementia diagnosis. Our results suggest that the substantial cost effects of dementia are mostly due to moderate/severe dementia and multiple comorbidities.

There are several limitations in the study. First, as noted before, it is important to emphasize that the sample in the study comes from an ethnically diverse, predominantly Hispanic and black population. Existing studies have shown that in the general population, as dementia progresses, individuals are more likely to be in nursing homes, and often spend down to Medicaid. However, in this study, Medicaid enrollment was higher in individuals with mild dementia. Hispanic caregivers have historically been less likely than non-Hispanic caregivers to place their loved one in nursing homes. A recent study reported nursing home admission among non-Hispanic blacks and Hispanics is far lower than non-Hispanic whites, particularly after health and socioeconomic characteristics taken into account [50]. Another study also showed that when they do, Hispanic and black minorities are more likely to be placed in nursing homes of poorer quality [51]. It is possible that the differences in Medicaid eligibility by dementia severity that we observed in the cohort may reflect some of these differences. Caring for minority elders is an important policy concern. Future studies will examine Medicaid use and nursing home placement in this cohort. The analysis data also included individuals who had Medicare coverage for the majority of months during each year of the study. Caution should be exercised in generalizing results from this study to other populations such as Medicare population as a whole, or the Medicare FFS population.

Second, the costs reported in this study only reflect Medicare costs and do not reflect the overall societal costs associated with dementia, including costs associated with informal caregiving, long term care costs which are not covered by Medicare, out-ofpocket costs, which also exert tremendous burden on the society, and could be differently affected by the potential interaction between severity and comorbidity. It should be noted that the relationship between Medicare costs and dementia severity and comorbidities are associations, not causations. Using total Medicare care cost as an outcome measure, it is difficult to separately identify healthcare use for dementia or for other comorbidities. A recent study showed that while non-dementia related healthcare use and costs were increased with levels of comorbidities, dementia related healthcare use and costs were similar across all levels of comorbidities [19]. In future studies, closer examination into the drivers of different types of cost are much needed.

Third, while accurate in-person clinical assessments were used to identify dementia and assess dementia severity in our study, presence of other conditions was obtained from Medicare claims and was not validated via clinical examinations. Misdiagnosis or miscoding of diagnoses, particularly in the presence of dementia, cannot be ruled out. However, while prevalence rates between studies are expected to vary because of heterogeneity of study settings, differences in the methods that conditions were ascertained, and differences in the demographic characteristics of the samples, our estimates of prevalence of comorbidities are in general comparable to those reported in the literature. Such errors in measurement in the level of comorbidities will likely lead to underestimation of their effects on costs, and our estimates of their effects may be conservative. Lastly, we used one method for quantifying the burden of multimorbidity. While the Elixhauser is widely used measure of comorbidities burden [52], there are other tools available. Our using a simple count of total number of comorbidities should be improved upon for measuring of complexity of illness to predict cost in future studies.

Older adults with dementia often have multiple comorbid conditions which are associated with a greater risk of dying, poor functional status, reduced quality of life and greater use of health care services and costs. By examining the role of dementia severity 
and individual as well as number of comorbidities, our work sheds light on the complex relationship between dementia and comorbidity and their impact on healthcare costs. It is important to realize that dementia-related costs will increase as dementia progresses to more severe stages even with no changes in comorbidities. However, as in normal aging, much of the healthcare cost in demented individuals is directly related to the comorbidities present. We must further examine how the presence of dementia influence the care individuals receive for their comorbidities to improve management of comorbidities. Policy makers should extend their focus on population of individuals with multiple comorbidities and dementia to improve care and reduce costs including the creation of more comprehensive programs that may be tailored to the complexity of care required for persons with dementia. Improving management of comorbidities will be increasingly important as we work to contain high costs of dementia care.

\section{ACKNOWLEDGMENTS}

This research was supported by grants from the National Institute on Aging (AG07370, AG037212). Dr. Zhu is also supported by the Department of Veterans Affairs, Veterans Health Administration. The views expressed in this article are those of the authors and do not necessarily represent the views of the Department of Veterans Affairs.

Authors' disclosures available online (http://j-alz. com/manuscript-disclosures/16-1077r2).

\section{SUPPLEMENTARY MATERIAL}

The supplementary material is available in the electronic version of this article: http://dx.doi.org/ 10.3233/JAD-161077.

\section{REFERENCES}

[1] Alzheimer's, Association (2015) 2015 Alzheimer's disease facts and figures. Alzheimers Dement 11, 332-384.

[2] Hurd MD, Martorell P, Delavande A, Mullen KJ, Langa KM (2013) Monetary costs of dementia in the United States. N Engl J Med 368, 1326-1334.

[3] Schubert CC, Boustani M, Callahan CM, Perkins AJ, Carney CP, Fox C, Unverzagt F, Hui S, Hendrie HC (2006) Comorbidity profile of dementia patients in primary care: Are they sicker? $J$ Am Geriatr Soc 54, 104-109.

[4] Doraiswamy PM, Leon J, Cummings JL, Marin D, Neumann PJ (2002) Prevalence and impact of medical comorbidity in Alzheimer's disease. J Gerontol A Biol Sci Med Sci 57, M173-M177.

[5] Hill JW, Futterman R, Duttagupta S, Mastey V, Lloyd JR, Fillit H (2002) Alzheimer's disease and related dementias increase costs of comorbidities in managed Medicare. Neurology $\mathbf{5 8}, \mathbf{6 2 - 7 0 .}$

[6] Centers for Disease Control, Prevention (2007) Prevalence of chronic kidney disease and associated risk factors-United States, 1999-2004. MMWR Morb Mortal Wkly Rep 56, 161165.

[7] Malone DC, McLaughlin TP, Wahl PM, Leibman C, Arrighi HM, Cziraky MJ, Mucha LM (2009) Burden of Alzheimer's disease and association with negative health outcomes. Am J Manag Care 15, 481-488.

[8] Barnes DE, Yaffe K (2011) The projected effect of risk factor reduction on Alzheimer's disease prevalence. Lancet Neurol 10, 819-828.

[9] Kuo TC, Zhao Y, Weir S, Kramer MS, Ash AS (2008) Implications of comorbidity on costs for patients with Alzheimer disease. Med Care 46, 839-846.

[10] Centers for Disease Control, Prevention (2011) Prevalence of Coronary Heart Disease, United States, 2006-2010. MMWR 60, 1377-1412.

[11] Yang Z, Zhang K, Lin PJ, Clevenger C, Atherly A (2012) A longitudinal analysis of the lifetime cost of dementia. Health Serv Res 47, 1660-1678.

[12] Zuliani G, Galvani M, Sioulis F, Bonetti F, Prandini S, Boari B, Guerzoni F, Gallerani M (2012) Discharge diagnosis and comorbidity profile in hospitalized older patients with dementia. Int J Geriatr Psychiatry 27, 313-320.

[13] Heun R, Schoepf D, Potluri R, Natalwala A (2013) Alzheimer's disease and co-morbidity: Increased prevalence and possible risk factors of excess mortality in a naturalistic 7-year follow-up. Eur Psychiatry 28, 40-48.

[14] Bauer K, Schwarzkopf L, Graessel E, Holle R (2014) A claims data-based comparison of comorbidity in individuals with and without dementia. BMC Geriatr 14, 10.

[15] Bunn F, Burn AM, Goodman C, Rait G, Norton S, Robinson L, Schoeman J, Brayne C (2014) Comorbidity and dementia: A scoping review of the literature. BMC Med $\mathbf{1 2}$, 192.

[16] Centers for Disease Control and Prevention (2014) National Diabetes Statistics Report: Estimates of Diabetes and Its Burden in the United States 2014. US Department of Health and Human Services, Atlanta, GA.

[17] Callahan CM, Schubert CC (2014) Dementia: The complexities of comorbidity in dementia. Nat Rev Neurol 10, 184-186.

[18] Smith T, Maidment I, Hebding J, Madzima T, Cheater F, Cross J, Poland F, White J, Young J, Fox C (2014) Systematic review investigating the reporting of comorbidities and medication in randomized controlled trials of people with dementia. Age Ageing 43, 868-872.

[19] Griffith LE, Gruneir A, Fisher K, Panjwani D, Gandhi S, Sheng L, Gafni A, Patterson C, Markle-Reid M, Ploeg J (2016) Patterns of health service use in community living older adults with dementia and comorbid conditions: A population-based retrospective cohort study in Ontario, Canada. BMC Geriatr 16, 177.

[20] Wolff JL, Starfield B, Anderson G (2002) Prevalence, expenditures, and complications of multiple chronic conditions in the elderly. Arch Intern Med 162, 2269-2276.

[21] Bynum JP, Rabins PV, Weller W, Niefeld M, Anderson GF, Wu AW (2004) The relationship between a dementia 
diagnosis, chronic illness, Medicare expenditures, and hospital use. J Am Geriatr Soc 52, 187-194.

[22] Friedman B, Jiang HJ, Elixhauser A, Segal A (2006) Hospital inpatient costs for adults with multiple chronic conditions. Med Care Res Rev 63, 327-346.

[23] Jonsson L, Wimo A (2009) The cost of dementia in Europe: A review of the evidence, and methodological considerations. Pharmacoeconomics 27, 391-403.

[24] Lochner KA, Goodman RA, Posner S, Parekh A (2013) Multiple chronic conditions among Medicare beneficiaries: State-level variations in prevalence, utilization, and cost, 2011. Medicare Medicaid Res Rev 3, pii: mmrr.003.03.b02.

[25] Taylor DH Jr, Schenkman M, Zhou J, Sloan FA (2001) The relative effect of Alzheimer's disease and related dementias, disability, and comorbidities on cost of care for elderly persons. J Gerontol B Psychol Sci Soc Sci 56, S285-S293.

[26] Newcomer R, Clay T, Luxenberg JS, Miller RH (1999) Misclassification and selection bias when identifying Alzheimer's disease solely from Medicare claims records. $J$ Am Geriatr Soc 47, 215-219.

[27] Fillit H, Geldmacher DS, Welter RT, Maslow K, Fraser M (2002) Optimizing coding and reimbursement to improve management of Alzheimer's disease and related dementias. J Am Geriatr Soc 50, 1871-1878.

[28] Lin PJ, Kaufer DI, Maciejewski ML, Ganguly R, Paul JE, Biddle AK (2010) An examination of Alzheimer's disease case definitions using Medicare claims and survey data. Alzheimers Dement 6, 334-341.

[29] Stern Y, Andrews H, Pittman J, Sano M, Tatemichi T, Lantigua R, Mayeux R (1992) Diagnosis of dementia in a heterogeneous population. Development of a neuropsychological paradigm-based diagnosis of dementia and quantified correction for the effects of education. Arch Neurol 49, 453-460.

[30] Scarmeas N, Levy G, Tang MX, Manly J, Stern Y (2001) Influence of leisure activity on the incidence of Alzheimer's disease. Neurology 57, 2236-2242.

[31] Chronic Condition DataWarehouse: Getting Started with CMS Medicare Administrative Research Files: A Technical Guidance Paper (2016) Available from: https://www.ccw data.org/web/guest/technical-guidance-documentation

[32] McKhann G, Drachman D, Folstein M, Katzman R, Price D, Stadlan EM (1984) Clinical diagnosis of Alzheimer's disease: Report of the NINCDS-ADRDA Work Group under the auspices of Department of Health and Human Services Task Force on Alzheimer's Disease. Neurology 34, 939-944.

[33] Morris JC (1993) The Clinical Dementia Rating (CDR): Current version and scoring rules. Neurology 43, 24122414.

[34] Diehr P, Yanez D, Ash A, Hornbrook M, Lin DY (1999) Methods for analyzing health care utilization and costs. Annu Rev Public Health 20, 125-144.

[35] Bureau of Labor Statistics, Consumer price index, http:// www.bls.gov/cpi/home.htm

[36] NIH NCI, SEER-Medicare: Calculation of Comorbidity Weights, https://healthcaredelivery.cancer.gov/seermedi care/program/comorbidity.html
[37] Quan H, Sundararajan V, Halfon P, Fong A, Burnand B, Luthi JC, Saunders LD, Beck CA, Feasby TE, Ghali WA (2005) Coding algorithms for defining comorbidities in ICD-9-CM and ICD-10 administrative data. Med Care 43, 1130-1139.

[38] Elixhauser A, Steiner C, Harris DR, Coffey RM (1998) Comorbidity measures for use with administrative data. Med Care 36, 8-27.

[39] Blessed G, Tomlinson BE, Roth M (1968) The association between quantitative measures of dementia and of senile change in the cerebral grey matter of elderly subjects. $\mathrm{Br} \mathrm{J}$ Psychiatry 114, 797-811.

[40] Richards M, Marder K, Bell K, Dooneief G, Mayeux R, Stern Y (1991) Interrater reliability of extrapyramidal signs in a group assessed for dementia. Arch Neurol 48, 11471149.

[41] Mullahy J (2009) Econometric modeling of health care costs and expenditures: A survey of analytical issues and related policy considerations. Med Care 47, S104-S108.

[42] Mihaylova B, Briggs A, O’Hagan A, Thompson SG (2011) Review of statistical methods for analysing healthcare resources and costs. Health Econ 20, 897-916.

[43] Manning W (2012) Dealing with skewed data on costs and expenditures, E. Elgar Publishing, Cheltenham.

[44] Albert SM, Glied S, Andrews H, Stern Y, Mayeux R (2002) Primary care expenditures before the onset of Alzheimer's disease. Neurology 59, 573-578.

[45] Zhu CW, Cosentino S, Ornstein K, Gu Y, Andrews H, Stern Y (2015) Use and cost of hospitalization in dementia: Longitudinal results from a community-based study. Int J Geriatr Psychiatry 30, 833-841.

[46] Tinetti ME, McAvay GJ, Chang SS, Newman AB, Fitzpatrick AL, Fried TR, Peduzzi PN (2011) Contribution of multiple chronic conditions to universal health outcomes. $J$ Am Geriatr Soc 59, 1686-1691.

[47] Salisbury C, Johnson L, Purdy S, Valderas JM, Montgomery AA (2011) Epidemiology and impact of multimorbidity in primary care: A retrospective cohort study. Br J Gen Pract 61, e12-e21.

[48] Barnett K, Mercer SW, Norbury M, Watt G, Wyke S, Guthrie B (2012) Epidemiology of multimorbidity and implications for health care, research, and medical education: A crosssectional study. Lancet 380, 37-43.

[49] Guthrie B, Payne K, Alderson P, McMurdo ME, Mercer SW (2012) Adapting clinical guidelines to take account of multimorbidity. BMJ 345, e6341.

[50] Thomeer MB, Mudrazija S, Angel JL (2015) How do race and Hispanic ethnicity affect nursing home admission? Evidence from the Health and Retirement Study. J Gerontol B Psychol Sci Soc Sci 70, 628-638.

[51] Fennell ML, Feng Z, Clark MA, Mor V (2010) Elderly hispanics more likely to reside in poor-quality nursing homes. Health Aff (Millwood) 29, 65-73.

[52] Yurkovich M, Avina-Zubieta JA, Thomas J, Gorenchtein M, Lacaille D (2015) A systematic review identifies valid comorbidity indices derived from administrative health data. J Clin Epidemiol 68, 3-14. 